\title{
Factors associated with non-adherence to the treatment of vivax malaria in a rural community from the Brazilian Amazon Basin
}

\author{
Eduardo Dias Almeida ${ }^{[1]}$ and José Luiz Fernandes Vieira ${ }^{[1]}$
}

[1]. Faculdade de Farmácia, Universidade Federal do Pará, Belém, Pará, Brasil.

\begin{abstract}
Introduction: We investigated the association between demographic and behavioral factors and non-adherence to antimalarial therapy. Methods: A demographic questionnaire and 5-item self-reported questionnaire regarding non-adherence were completed by 135 patients after treatment for Plasmodium vivax. Results: Treatment interruption, but not demographic factors, was significantly associated with non-adherence to therapy. The likelihood of non-adherence was 5.16 times higher when the patients felt better than when they felt worse. The relative risk of parasitic resurgence was 3.04 times higher in non-adherent patients. Conclusions: Treatment interruption is significantly associated with treatment adherence.
\end{abstract}

Keywords: Malaria. Resurgence. Adherence.

The failure of chloroquine and primaquine to treat malaria by Plasmodium vivax has recently increased worldwide, including in the Brazilian Amazon Basin, where the treatment is not effective for an estimated $4-15 \%$ of patients $^{(1)(2)(3)}$. Parasitic resurgence after treatment completion is commonly associated with resistance of $P$. vivax strains against antimalarial drugs, the relapse of hypnozoites, reinfection in endemic areas, or inadequate exposure to antimalarial drugs, which can be the result of low-quality drugs, incorrect dosage, drug interactions, vomiting or other gastrointestinal disorders, and non-adherence to the prescribed regimen ${ }^{(4)(5)(6)}$.

The latter is a challenge for the control and eradication of the disease, because the exposure of the parasite to subtherapeutic concentrations of antimalarial drugs can lead to infection recrudescence and the selection of resistant strains. Non-adherence has been associated with demographic and behavioral variables, such as education level, gender, age, level of knowledge about the disease and its prevention, adverse effects, poor interaction between patients and their dispensers, loss of drugs, perceived ineffectiveness of the drug, and difficulty accessing health facilities ${ }^{(4)(5)(6)}$.

In a previous study that aimed to validate a 5-item selfreported questionnaire for the estimation of non-adherence to antimalarial drugs, $33.2 \%$ of the participants in the riverside community of the Amazon Basin were non-adherent; however, the factors responsible for the non-adherence were not

Corresponding author: Dr. José Luiz Fernandes Vieira.

e-mail: jvieira@ufpa.br

Received 7 August 2015

Accepted 23 December 2015 determined $^{(7)}$. To improve disease control and eradication efforts, it would be helpful to understand the causes of non-adherence ${ }^{(5)}$; therefore, the current study aimed to evaluate the association between non-adherence and demographic and behavioral factors and to estimate the risk of parasitic resurgence in non-adherent patients during the follow-up period.

A prospective study was conducted from September to October 2012 at the Basic Unit Health in Anajas, State of Pará, a rural municipality on Marajo Island (059'21' $\mathrm{S}$ and $\left.49^{\circ} 56^{\prime} 24^{\prime \prime} \mathrm{W}\right)$, where the transmission of malaria is intense, with some seasonal fluctuations throughout the year. We randomly recruited adults with slide-confirmed infection by $P$. vivax among those who spontaneously sought care in the health facilities of the municipality. Patients with mixed malaria or signs and symptoms of severe malaria, parasite density $>5 \%$, glucose-6-phospate dehydrogenase deficiency, or previous treatment with antimalarial drugs in the prior 4 weeks were excluded. The required sample size was estimated at 135 patients, based on the assumption of $13 \%$ non-adherence, with a significance level of $95 \%$ and precision level of $5 \%$.

Each subject was administered multiple oral doses of chloroquine $(10 \mathrm{mg} / \mathrm{kg}$ on day 0 and $7.5 \mathrm{mg} / \mathrm{kg}$ on days 1 and 2 ) co-administered with primaquine $(0.50 \mathrm{mg} / \mathrm{kg} / \mathrm{d}$ for 7 days, as 13.2-mg primaquine phosphate tablets) ${ }^{(8)}$. The primaquine dose was adjusted by body weight. The community health workers were responsible for prescribing and dispensing the antimalarial drugs under the supervision of the medical staff. Patients received specific instructions about daily self-administration and were strongly advised to complete the course of treatment.

Data were collected by two trained research assistants, who conducted the interview and collected the structured 5-item selfreported questionnaire, which was based on the original 4-item 
questionnaire by Morisky and previously validated by the research team ${ }^{(7)(9)}$; informed consent forms; and demographic questionnaire from all patients. Patients were monitored daily, and those who completed the 7-day treatment were visited at their homes for the interview, during which the following information was collected: area of residence, age, parasitemia at admission, gender, and number of days after symptom onset that treatment was sought.

In the current study, adherence was defined as abiding by the recommended dose and period of antimalarial drugs and was estimated from the responses (yes/no) to the 5-item selfreported questionnaire: I) Did you ever forget to take your pills? II) Were you ever careless in taking your pills? III) Did you ever miss your pills when you were feeling better? IV) Did you ever miss any of your pills because you were feeling sick? V) Did you taking more than one the dose when you were feeling sick? A response of no was scored as 1, and a response of yes was scored as 0 . Levels of adherence to antimalarial therapy were determined by simply adding the items, with total scores ranging from 0 to 5 points. Patients with scores below the median score were classified as non-adherent ${ }^{(7)(9)}$.

Patients were followed up by passive surveillance at the health facility for a period of 180 days, and any further episodes of malaria were confirmed by microscopy and underwent the same course of treatment as at enrolment. Also, records from the National Malaria Database [Sistema de Informação de Vigilância Epidemiológica-Malária (SIVEP-Malaria)] were searched to identify episodes of malaria for any of the enrolled patients.

Data are expressed as percentages, median, and 95\% confidence intervals (CIs). Chi-square tests were used to compare the demographic variables as well as variables based on treatment interruption. The frequencies of yes responses to any of the 5 questions were compared. Logistic regression analysis was used to estimate the associations between adherence and demographic or behavioral characteristics as well as the association between adherence behavior and resurgence of parasites. Data were analyzed using Statistical Package for the Social Sciences (SPSS) software, Release 21 (IBM Inc., Armonk, NY, USA). The accepted significance level was 5\%.

The mean age of the patients was $29 \pm 12$ years (range, 18-75 years), and the majority of patients were men $(60.7 \%$; $95 \% \mathrm{CI}$, 52.6-67.4). Of the 135 patients, 66 lived in rural areas (48.9\%; 95\% CI, 40.0-56.3).

The parasite density on admission was similar between adherent $(974 \pm 7.7)$ and non-adherent $(864 \pm 7.6)$ patients. There were no significant associations between adherence behaviors and gender, age, zone of residence, and the number of days after symptom onset that treatment was sought.

Compared with all of the other questions, yes responses were given significantly more often for the questions related to treatment interruption when patients felt better or worse (Figure 1). There were significant associations between nonadherence behavior and treatment interruption when the patient felt better or worse due to the medication. The odds of nonadherence was 5.16 times higher for patients who abandoned treatment when they felt better than for patients who abandoned treatment when they felt worse due to medication (Table 1).
During the 180-day follow-up period, there were $19(14.1 \%)$ cases of resurgence of parasites, at 1-2 weeks ( 8 cases) and 8-19 weeks (11 cases) post-treatment. The relative risk of parasitic resurgence in non-adherent patients was 3.04 higher than in adherent patients (odds ratio, 3.95; 95\% CI, 1.31-11.98; $\mathrm{p}=0.0071)$ (Table 2).

The rate of non-adherence in the current study corroborates previous reports in other areas of the Amazon Basin, as in Ecuador, where the rate of non-adherence was $34.1 \%$ in patients under the same therapeutic regimen ${ }^{(10)}$. In Brazil, the majority of malaria cases occur in the Amazon region, where geographic, economic, and social variables create challenges for disease control and eradication. The native population has low educational levels and limited knowledge about the disease and its prevention. Each inhabitant has his/her own perception about the disease based on their experiences with previous episodes of malaria, the frequencies of these episodes, the intensity of signs and symptoms, and the use and effectiveness of antimalarial drugs ${ }^{(11)}$.

Interestingly, demographic factors, such as age and gender, were not associated with adherence to the antimalarial therapy. The majority of the patients were of working age, suggesting a potential association between malaria and occupational activities in this endemic area. The influence of age on nonadherence is inconclusive, although children and elderly patients are generally more adherent to prescribed regimens ${ }^{(12)}$. Both genders were equally affected by the disease, and a similar proportion of each gender was non-adherent to the prescribed regimen. Conversely, a previous report found a higher rate of non-adherence for men ${ }^{(13)}$, which might be explained by the baseline characteristics of the patients in the current study.

The area of residence, number of days after symptom onset that treatment was sought, and parasitemia at admission did not influence adherence to the prescribed regimen. The patients in the current study were recruited from both rural and urban

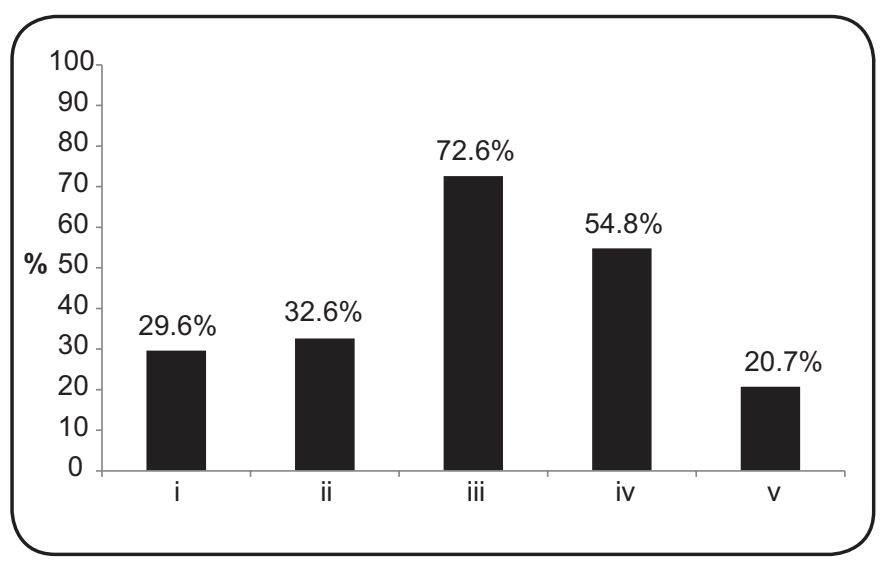

FIGURE 1 - Percentage of yes responses to each of the 5 items of the self-reported questionairre that collected information about adherence to antimalarial treatment. I: Did you ever forget to take your pills? II: Were you ever careless in taking your pills? III: Did you ever miss your pills when you were feeling better? IV: Did you ever miss any of your pills because you were feeling sick? V: Did you replicate the dose when you were feeling sick? 
TABLE 1 - Treatment interruption, based on treatment adherence by patients undergoing treatment for Plasmodium vivax.

\begin{tabular}{|c|c|c|c|c|c|c|c|c|}
\hline \multirow[b]{2}{*}{ Questions } & \multicolumn{2}{|c|}{$\begin{array}{l}\text { Non-adherent } \\
\quad(\mathrm{n}=42)\end{array}$} & \multicolumn{2}{|c|}{$\begin{array}{c}\text { Adherent } \\
(\mathrm{n}=93)\end{array}$} & \multicolumn{2}{|c|}{$\begin{array}{c}\text { Total } \\
(n=135)\end{array}$} & \multirow{2}{*}{$\begin{array}{c}\text { OR } \\
(95 \% \mathrm{CI})\end{array}$} & \multirow[b]{2}{*}{ p-value } \\
\hline & $\mathbf{n}$ & $\%$ & $\mathbf{n}$ & $\%$ & $\mathbf{n}$ & $\%$ & & \\
\hline yes & 41 & 41.8 & 57 & 55.2 & 98 & 72.6 & 25.9 & \\
\hline no & 1 & 2.7 & 36 & 7.3 & 37 & 27.4 & $(3.4-196.6)$ & \\
\hline yes & 33 & 44.6 & 41 & 55.4 & 74 & 54.8 & 4.7 & \\
\hline no & 9 & 14.8 & 52 & 85.2 & 61 & 45.2 & $(2.0-10.8)$ & \\
\hline
\end{tabular}

OR: odds ratio; CI: confidence interval.

TABLE 2 - Relative risk of a new episode of disease, according to adherence behavior to the antimalarial treatment.

\begin{tabular}{|c|c|c|c|c|c|c|c|c|}
\hline Adherence behavior & \multicolumn{2}{|c|}{ With recurrence } & \multicolumn{2}{|c|}{ Without recurrence } & $\begin{array}{c}\text { Total } \\
\mathbf{n}\end{array}$ & RR & $95 \% \mathrm{CI}$ & p-value \\
\hline Non-adherent & 11 & $26.2(14.3-35.7)$ & 31 & $73.8(57.2-83.3)$ & 42 & 3.04 & $1.32-7.01$ & 0.0071 \\
\hline Adherent & 8 & $8.6(4.3-11.8)$ & 85 & $91.4(83.9-95.7)$ & 93 & & & \\
\hline Total & 19 & $14.1(8.9-18.5)$ & 120 & $88.9(83.7-92.6)$ & 135 & & & \\
\hline
\end{tabular}

RR: relative risk; CI: confidence interval.

areas, and the distance from the small rural properties to the health facilities was approximately $1-2 \mathrm{~km}$. New cases of malaria in rural communities are regularly and actively searched by medical staff of the municipality. Furthermore, the low parasite density in both groups is generally associated with mild signs and symptoms of the disease.

However, human behavior, in terms of decision-making, was related with non-adherence to the prescribed regimen. The patients responded that they did interrupt their treatment when they felt better or worse with the medication, which supports the findings of previous studies regarding medication use ${ }^{(4)(5)}$. Also, the probability of a patient becoming non-adherent due to treatment interruption with improvement was approximately 5 times higher than becoming non-adherent due to treatment interruption because they felt worse. Parasite clearance, which occurs 48-72 after treatment initiation for Brazilian strains of $P$. vivax, is accompanied by the cessation of disease signs and symptoms ${ }^{(2)(3)(11)(13)}$. After this period, patients feel better and do not consider themselves ill, which is usually accompanied by a prompt return to work and abandonment of the treatment. This behavior could be considered the major determinant for non-adherence in this municipality. Although the side effects with antimalarial drug use can promote dissatisfaction with the treatment, the probability of a patient becoming nonadherent because they worsened after antimalarial drug use is low, because the side effects with therapeutic doses of chloroquine and primaquine are mild and usually related with the gastrointestinal system, including vomiting and nausea ${ }^{(6)(7)(8)}$.
The main limitations of the current study were that it was conducted at a single health facility over a relatively short period, which may not be representative of other municipalities in the same region or the annual pattern. In addition, participation in a follow-up study could alter adherence behavior. Moreover, there are some shortcomings with using self-reported methods to estimate adherence, because patients might tend to overestimate adherence and underestimate non-adherence ${ }^{(4)(5)(7)(8)(10)}$.

The resurgence of parasites was reported in 19 patients during the follow-up period; however, the causes of resurgence, such as resistance, relapse, a new infection, or incorrect drug exposure, were not assessed. In Brazil, the relapse of $P$. vivax occurs during the first 6 months post-treatment, but long-latency forms might coexist with frequent relapse phenotypes ${ }^{(14)}$. Two patterns of resurgence were found in the current study: short duration (1-2 weeks) and long duration (8-19 weeks). In both, the relative risk of a new episode of malaria in non-adherent patients was 3.04 times higher than in adherent patients, supporting the findings of a previous study in which low adherence to treatment was an important predictor of a subsequent episode of malaria in the same individual ${ }^{(15)}$.

\section{Ethical considerations}

The Ethical Committee of the Evandro Chagas Institute reviewed and approved this study (Comitê de Ética em Pesquisa/ Instituto Evandro Chagas/Secretaria de Vigilância em Saúde/ Ministério da Saúde 0028/2010). Patients were informed of the study purpose and provided written consent before participating in the interview. 


\section{CONFLICT OF INTEREST}

The authors declare that there is no conflict of interest.

\section{REFERENCES}

1. World Health Organization. World Malaria Report. Geneva: 2013.

2. Alecrim MGC, Alecrim W, Macedo V. Plasmodium vivax resistance to chloroquine (R2) and mefloquine (R3) in Brazilian Amazon region. Rev Soc Bras Med Trop 1999; 32:67-68.

3. De Santos Filho FS, Arcanjo AR, Chehuan YM, Costa MR, Martinez-Espinosa FE, Vieira JL, et al. Chloroquine-resistant Plasmodium vivax, Brazilian Amazon. Emerg Infect Dis 2007; 13:1225-1226.

4. Yeung S, White NJ. How do patients use antimalarial drugs? A review of the evidence. Trop Med Int Health 2005; 10:121-138.

5. Bruxvoort K, Goodman C, Kachur SP, Schellenberg D. How patients take malaria treatment: a systematic review of the literature on adherence to antimalarial drugs. PLoS One 2014; 9:e84555. doi:10.1371/journal.pone.0084555.

6. Hill DR, Baird JK, Parise ME, Lewis LS, Ryan ET, Magill AJ. Primaquine: report from $\mathrm{CDC}$ expert meeting on malaria chemoprophylaxis. Am J Trop Med Hyg 2006; 65:402-415.
7. Almeida ED, Rodrigues LCS, Vieira JLF. Estimates of adherence to treatment of vivax malaria. Malar J 2014; 13-321-327.

8. World Health Organization. Guidelines for the treatment of malaria. Geneva: 2015 .

9. Morisky DE, Green LW, Levine DM. Concurrent and predictive validity of a self-reported measure of medication adherence. Med Care 1986; 24:67-74.

10. Yépez MC, Zambrano D, Carrasco F, Yépez RF. Factores asociados con el incumplimiento del tratamiento antipalúdico en pacientes ecuatorianos. Rev Cubana Med Trop 2000; 52:81-89.

11. Barreto ML, Teixeira MG, Bastos FI, Ximenes RAA, Barata RB, Rodrigues LC. Successes and failures in the control of infectious diseases in Brazil: social and environmental context, policies, interventions, and research needs. Lancet 2011; 377:1877-1889.

12. Krause G, Sauerborn R. Comprehensive community effectiveness of health care. A study of malaria treatment in children and adults in rural Burkina Faso. Ann Trop Paediatr 2000; 20:273-282.

13. Pereira EA, Ishikawa EAY, Fontes CJF. Adherence to Plasmodium vivax malaria treatment in the Brazilian Amazon Region. Malar J 2011; 10:355-361.

14. Tauil PL, Luz FC, Oliveira AP, Deckers FA, Santos JB. Vivax malaria with long incubation period, detected in the Federal District: three case reports. Rev Soc Bras Med Trop 2010; 43:213-214.

15. Duarte EC, Gyorkos TW. Self-reported compliance with last malaria treatment and occurrence of malaria during follow-up in a Brazilian Amazon population. Trop Med Int Health 2003; 8:518-524. 